\title{
Gradhiva
}

GRADHI

Revue d'anthropologie et d'histoire des arts

$21 \mid 2015$

Création plastique d'Haïti

\section{La diaspora en dialogue : James A. Porter et Loïs Mailou Jones Pierre-Noël, ou comment écrire l'histoire de l'art haïtien}

Developing Diasporic Dialogues: James A. Porter, Loïs Mailou Jones Pierre-Noël and the Writing of Haitian Art History

\section{Lindsay J. Twa}

Traducteur : Claire Duvivier

\section{(2) OpenEdition}

Journals

Édition électronique

URL : http://journals.openedition.org/gradhiva/2933

DOI : 10.4000/gradhiva.2933

ISSN : 1760-849X

Éditeur

Musée du quai Branly Jacques Chirac

Édition imprimée

Date de publication : 1 février 2015

Pagination : 48-75

ISBN : 978-2-35744-075-3

ISSN : 0764-8928

Référence électronique

Lindsay J. Twa, « La diaspora en dialogue : James A. Porter et Loïs Mailou Jones Pierre-Noël, ou

comment écrire l'histoire de l'art haïtien », Gradhiva [En ligne], 21 | 2015, mis en ligne le 01 février 2015, consulté le 19 avril 2019. URL : http://journals.openedition.org/gradhiva/2933 ; DOI : 10.4000/ gradhiva.2933 
$2 \pi$
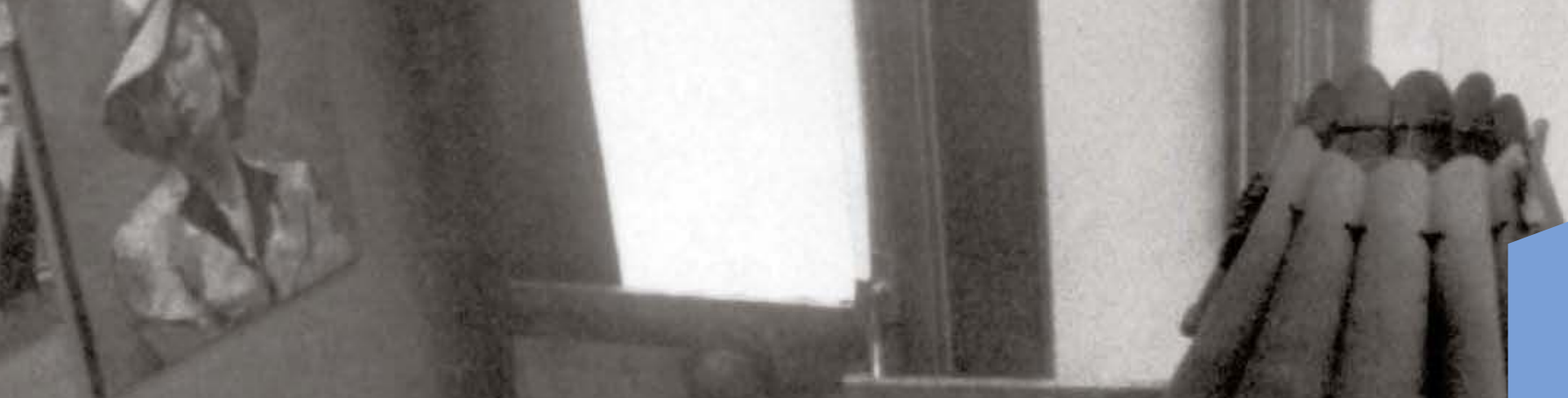


\section{La diaspora en dialogue: James A. Porter et Loïs Mailou Jones Pierre-Noël,}

ou comment écrire l'histoire de l'art haïtien

par Lindsay J. Twa

Jusqu'à une date récente, les historiens de l'art américains spécialistes de l'art haiitien - collectionneurs et artistes - développèrent leur réflexion en dehors des cercles académiques. Dans un premier temps, l'histoire de l'art haïtien fut donc élaborée dans un cadre intellectuel particulier par des individus dont les goûts et les intérêts personnels déterminèrent la nature des questions qui surgirent du dialogue avec les artistes haïtiens et leurs œuvres. Un exemple remarquable est celui de Selden Rodman, dont les commentaires exubérants sur l'art haiitien, conformes néanmoins au canon primitiviste, dominèrent la discipline pendant des dizaines d'années. Cet article examine les projets de recherche de James Amos Porter et Loïs Mailou Jones Pierre-Noël, artistes et historiens de l'art afro-américains. Leurs archives contiennent des éléments très fructueux permettant de remettre en question les analyses développées au milieu du xxe siècle sur l'art haïtien contemporain. Ils promurent tous deux la diversité des styles haïtiens ainsi qu'une longue liste d'artistes méconnus. Ils contribuèrent ainsi à élargir l'horizon du canon de l'art haïtien et à lutter contre les stéréotypes raciaux qui servaient alors à renforcer le statut inférieur des Noirs et à limiter, de manière générale, les droits des individus d'origine africaine. 


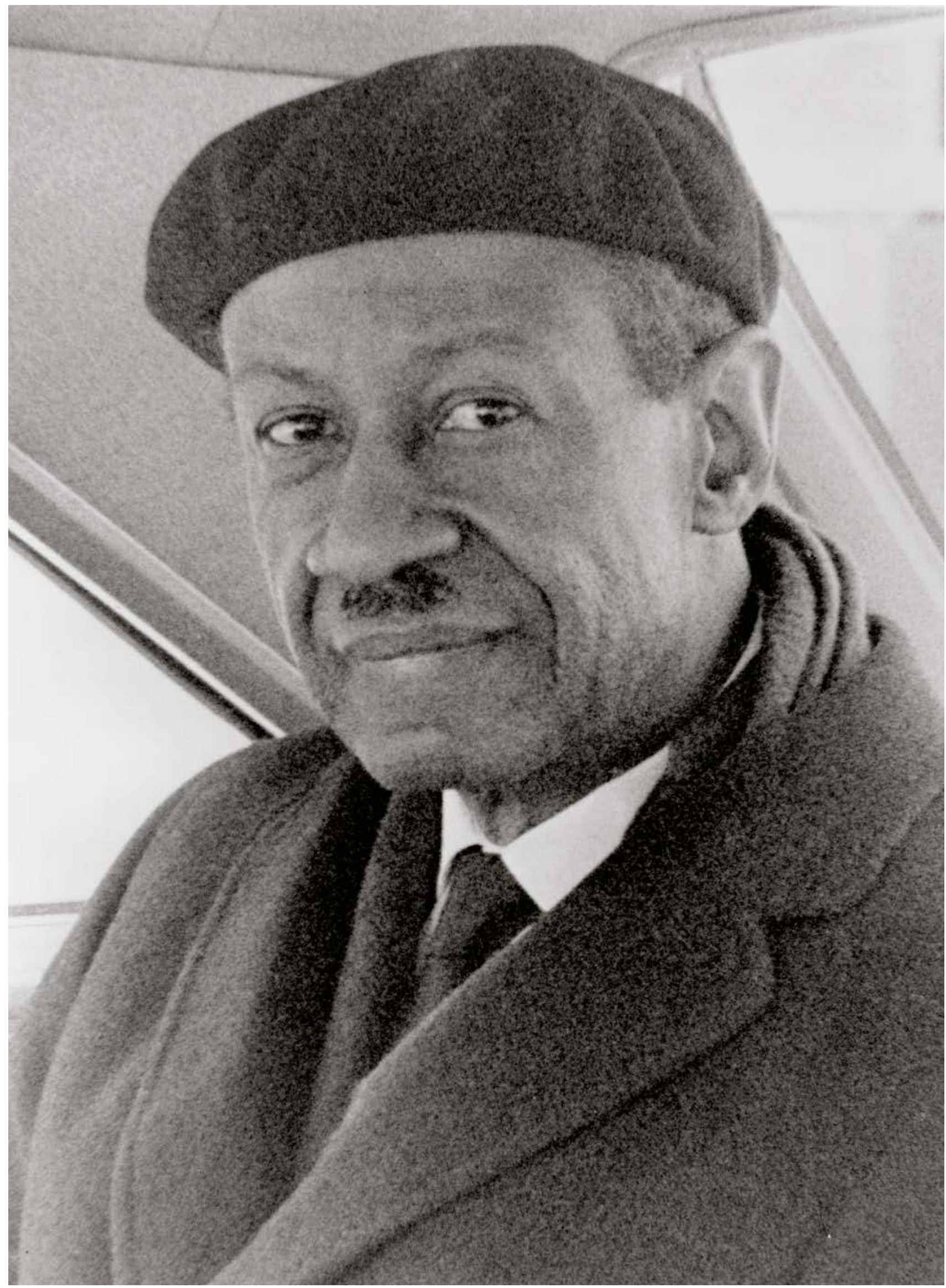


Au sein du marché de l'art mondial et des histoires de l'art qui y sont associées, l'art haïtien est un domaine relativement récent. Dans sa réception nord-américaine, en particulier, cette étude a été largement entreprise au niveau personnel, c'est-à-dire avec les marchés locaux, les mécènes et les artistes-chercheurs qui ont généralement opéré en dehors des limites académiques formelles. Par bien des aspects, I'histoire de l'art haïtien est semblable à celle des autres domaines impliquant des artistes marginalisés, depuis l'art populaire et l'«outsider art» jusqu'au champ en pleine croissance et en pleine maturation qu'est celle de l'art afro-américain. En comparant l'état de l'étude de l'art haïtien à ces autres domaines relativement récents, on distingue plusieurs similarités. Avant tout, il y a le besoin urgent de base documentaire: biographies et déclarations d'artistes, emplacement et qualité des œuvres d'art, histoire sociale des œuvres et de leurs sujets, etc. Des espaces plus spécialisés de théorisation - des mécanismes institutionnels et des structuralismes concomitants, ainsi que diverses autres théories critiques postmodernes - commencent seulement à être appliqués à ce domaine. Une grande partie du travail "de base», néanmoins, reste encore à faire - et il est rendu plus urgent encore par la destruction, lors du tremblement de terre de janvier 2010, d'institutions, d'archives et d'œuvres d'art. Dans ce besoin crucial de poursuivre la constitution d'une base documentaire biographique, iconographique et esthétique des œuvres, des concours de circonstance se produisent. Autrement dit, l'histoire est profondément façonnée par les coïncidences, les relations personnelles et les discussions. Ou ce que LeGrace Benson, une contributrice importante dans l'étude de l'art haïtien, a intelligemment décrit comme un crucial «réseau d'actes et de paroles et d'écrits» qui prouve que «les discussions [sont] l'infrastructure de la construction d'une nation» (Benson 1997-1998: 50).

Benson avait choisi ces mots pour évoquer le rôle catalyseur de Jean Price-Mars (1876-1969) dans la revalorisation de la culture populaire et de I'héritage haïtiens. Ceux-ci sont tout aussi pertinents pour évoquer le travail sur l'histoire de l'art dans la diaspora noire, ainsi que les œuvres intellectuelles et les textes d'accompagnement nécessaires à la construction de la connaissance du monde de l'art et de ses marchés, et à la valorisation et à la documentation des artistes et de leurs œuvres. En d'autres termes, derrière les théories et les métarécits, il y a d'abord les discussions locales. Comme Benson, il nous faut nous souvenir que le dialogue est l'infrastructure qui génère la connaissance, et c'est particulièrement vrai pour l'histoire de l'art haïtien du milieu du $x x^{e}$ siècle. Dans ce bref article, je veux explorer la structuration et le développement de cette étude pour la période s'étendant des années 1940 aux années 1970 grâce à un réseau de discussions localisées sur les aspirations de la diaspora noire mondiale centrées autour d'artistes afro-américains qui sont aussi devenus des chercheurs, plus spécifiquement James Amos Porter et Loïs Mailou Jones Pierre-Noël. En suivant ces deux fils directeurs, je veux clarifier certains des mécanismes qui se répercutent sur les écrits du milieu du $x x^{e}$ siècle concernant l'art haïtien.

Un corpus en expansion de discussions académiques sur la diaspora noire est fondé sur l'idée que cette circulation d'idées et d'héritages est un moyen de comprendre l'art moderne noir. Cela vaut autant pour l'étude de l'art haïtien que pour celle de la culture visuelle afro-américaine, et les deux

\section{ci-contre}

fig. 1

Photographie de James Amos Porter, s.d. James Amos Porter Papers, Robert W. Woodruff

Library, Emory University, Atlanta, GA, Manuscript, Archives \& Rare Book Library. (C) Photo Milan Uzelac (C) James Amos Porter Estate. 
champs d'investigation luttent pour déterminer quels éléments sont de «pure» origine africaine et quelles contributions sont des acculturations de source «blanche» européenne. Dans le même temps, les dialogues diasporiques présents dans l'art haïtien et l'art afro-américain connaissent au milieu du $x x^{e}$ siècle les mêmes difficultés et les mêmes interrogations: les rencontres directes avec l'Afrique étaient limitées et, le plus souvent, se bornaient à des discussions de seconde main. Comme le célèbre historien de l'art haïtien Michel-Philippe Lerebours l'a expliqué: «En ce qui concerne l'Afrique, son nom résonnait partout, mais [...] sans cette volonté de réelle communion avec des frères victimes du colonialisme, sans même cette réelle croyance dans les valeurs et les destinées du peuple d'origine africaine. » (Lerebours 1992: 712) C'étaient le cadre intellectuel et les objectifs de PriceMars dans son essai révolutionnaire Ainsi parla l'oncle (1928). La récupération et la revalorisation d'un héritage africain ancien sont également inscrites dans l'histoire du mouvement New Negro (connu aussi sous le nom de Renaissance de Harlem) aux États-Unis, qui adoptait l'idée d'une Afrique symbolique comme moyen de combattre le nihilisme social et culturel ressenti par les Afro-américains au sein des structures racistes des États-Unis. Se revendiquer fièrement de l'Afrique et se tourner vers des sources africaines était, selon les mots de l'artiste afro-américain et historien de l'art David Driskell, une «zone de confort investie racialement» (Driskell 2013). Tandis que beaucoup d'artistes concevaient et idéalisaient leur travail comme étant en résonance avec une Afrique ancestrale sacrée et héroïque, ce que nombre d'entre eux produisaient étaient en fait des explorations et des expressions des réalités contemporaines du Nouveau Monde.

\section{James A. Porter et The Black Artist}

De bien des façons, l'Afro-américain James Amos Porter (1905-1970) est venu en Haïti à la recherche de l'Afrique (fig. 1). II était peintre et historien de l'art, et son essai Modern Negro Art (1943) a fait date dans l'histoire de l'art afro-américain. L'approche qu'il avait de la recherche et de l'écriture en histoire de l'art était celle d'un historiographe formaliste. En tant que pionnier dans son domaine, son travail a été un travail de récupération et de documentation dans le but exprès de souligner le talent des artistes d'origine africaine et le fait qu'ils ont toujours été oubliés et négligés par les études d'histoire de l'art classiques. Dans le même temps, quand il parlait d'œuvres d'art, que ce soit dans ses écrits ou dans ses cours importants et influents à la Howard University de Washington, il insistait pour placer celles-ci au cœur de discussions sur une histoire visuelle plus large. II voulait que ses lecteurs et ses étudiants comprennent les «qualités durables » de l'art des artistes noirs comme faisant partie d'une histoire des relations et des discussions avec des mouvements d'art européen ou avec la production artistique africaine. II rejetait ce qu'il appelait une approche ethnographique, qui s'intéresse à la biographie des artistes et à leurs intentions (Porter 1992 XIII). II préférait employer l'historiographie comme une mesure défensive afin que le public comprenne d'abord la valeur et la qualité intrinsèques d'une œuvre d'art, puis remarque ensuite qu'elle est la création d'un artiste noir.

Après avoir publié ce qui deviendra le texte fondateur de l'histoire de l'art afro-américain en 1943, Porter a compris le besoin d'étendre son champ d'étude pour inclure le reste de la diaspora noire du Nouveau Monde. 
Il a reçu une subvention du General Education Board ${ }^{\mathbf{1}}$ pour rencontrer et étudier des artistes noirs en Haïti, à Cuba et dans le sud des États-Unis pendant l'année universitaire 1945-1946. Si son projet initial concernait la récupération et la célébration d'œuvres, son nouveau projet de recherche visait la construction de dialogues avec la diaspora noire.

Dans son dossier de demande de subvention au General Education Board, Porter expliquait qu'une année de voyage et d'études l'aiderait à améliorer «les relations raciales et interculturelles dans l'hémisphère occidental» (Porter 1945a). En proposant d'étudier «la contribution du Nègre aux arts vivants (peinture, sculpture et arts graphiques) à Cuba, en Haïti et aux États-Unis », il envisageait plusieurs résultats spécifiques supplémentaires. Premièrement, il voulait enquêter sur les œuvres contemporaines des artistes noirs et celles avec «le Nègre comme sujet». II se laissait ainsi la possibilité d'étudier des artistes d'origine hispanique ou amérindienne si leurs œuvres représentaient des Noirs. Deuxièmement, il soutenait que rassembler de tels matériaux de recherche lui permettrait de proposer de nouveaux cours sur «le rôle du Nègre en Amérique latine». Comme soussection de ce deuxième résultat envisagé, il arguait également que son étude «déterminerait l'efficacité des artistes nègres à Cuba et en Haïti en tant qu'interprètes des traditions et des idéaux des peuples cubain et haïtien de toutes les couleurs ". Ce qu'il espérait trouver en Haïti refléterait l'évolution des artistes haïtiens vers la représentation de sujets haïtiens, en particulier des scènes de la vie quotidienne, de l'histoire et de la religion populaire haïtienne, le vodou. Le troisième résultat attendu se concentrait spécifiquement sur Haïti et les mécanismes de son marché de l'art, que ce soit pour la vente des œuvres ou le soutien aux artistes. Porter a mis au jour une pénurie d'écrits sur l'art en Haïti, alors même que d'autres pans de la culture haïtienne (littérature, religion et musique) avaient été surdéterminés aux États-Unis par des écrits «d'intérêt touristique populaire». II prévoyait de prendre et de collecter des photographies et des diapositives de peintures, de sculptures et d'art graphique dans l'idée qu'elles serviraient de ressources significatives pour les étudiants et les chercheurs ne pouvant faire le voyage. Des rencontres avec des artistes étaient prévues dans leurs ateliers, en plus de visites aux musées, galeries et collections privées. Porter a aussi indiqué qu'il comptait s'entretenir avec des autorités, historiens et autres chercheurs éminents familiarisés avec les champs culturels de Cuba et Haïti (Porter 1945b).

Ces recherches devaient, en plus de développer la matière et les sujets pour ses cours à la Howard University, avoir pour résultat un «ouvrage bien documenté » qui serait une expansion diasporique noire de sa première étude de l'histoire de l'art afro-américain. Reprenant le format de Modern Negro Art, il prévoyait de documenter l'histoire des artistes noirs à Cuba et en Haïti, des plus anciens jusqu'aux plus contemporains. Mais, contrairement à ce qu'il avait fait dans sa première monographie, il considérait que le but ultime de ce projet était de présenter des analyses comparatives interculturelles de ces créateurs avec des artistes afro-américains, et de les analyser afin d'expliquer quels aspects provenaient de survivances culturelles africaines et quels autres étaient des créations ou syncrétismes du Nouveau Monde (ibid.). De cette façon, ses recherches s'alignaient avec
1. Fondation créée par John D. Rockefeller en 1902 afin de promouvoir l'éducation sans distinction de race, de sexe ou de religion. 


\section{ci-contre}

fig. 2

Croquis de James Amos Porter. James Amos Porter Papers, Robert W. Woodruff Library, Emory University, Atlanta, GA, Manuscript, Archives \& Rare Book Library. ( ) James Amos Porter Estate. celles de nombreux anthropologues, sociologues et travailleurs culturels des années 1930 et 1940 qui voyaient la documentation de survivances africaines dans le Nouveau Monde non seulement comme un moyen de décrire les talents et les réussites des Noirs, mais aussi comme un mécanisme pour combattre le racisme et les stéréotypes dominants qui imposaient l'idée de l'infériorité noire.

Porter est arrivé à Cuba en septembre 1945, passant, de son propre aveu, trois mois et dix-neuf jours dans le pays avant de se rendre en Haïti où il a séjourné deux mois et trois jours, de décembre 1945 à février 1946. Son séjour en Haïti a coïncidé avec celui du peintre cubain Wifredo Lam, dont les œuvres surréalistes se sont révélées une grande inspiration pour les artistes haïtiens, et avec la visite capitale d'André Breton qui a aidé le public haïtien à interpréter les peintures de Lam, et dont les conférences sur l'art et la littérature surréalistes et sa praxis révolutionnaire ont embrasé la jeune avant-garde haïtienne qui appelait à la rébellion (Porter 1946a). La présidence déjà tendue d'Élie Lescot est tombée en janvier 1946. Porter se trouvait donc en Haïti, pour documenter ses mouvements artistiques contemporains, à une époque extraordinaire.

À l'automne 1946, il a publié un article consacré à Haïti dans le journal afro-américain Opportunity. Son texte comprend une critique de la nature essentialisante de l'usage des paysans haïtiens comme simples icônes pittoresques alors que des artistes aussi bien haïtiens qu'étrangers les prenaient de plus en plus comme sujets centraux. L'article s'ouvre sur ses premières impressions d'Haïti, décrites en détail et avec élégance, depuis son arrivée à l'aéroport de Port-au-Prince et sa traversée des quartiers environnants jusqu'à son hôtel sur le Champ-de-Mars. Porter insiste ensuite pour se corriger lui-même (ainsi que le lecteur): «ll est somme toute trop facile de tomber dans cet impair esthétique si commun en présence du nouveau et de l'étrange, en d'autres termes de dire de la vie ou des paysages en Haïti: "Oh, comme c'est pittoresque." " II conteste les descriptions courantes d'Haïti comme endroit pittoresque et les condamne en tant que vision touristique simpliste du pays. "De l'intérieur, la vie haïtienne est fascinante mais pas pittoresque.» (Porter 1946b: 179) Ou plutôt, la "vue de l'intérieur" révèle non seulement la nature travailleuse des individus haïtiens, mais aussi les «limitations physiques et économiques» du pays. Porter soutient que l'extrême pauvreté du pays explique beaucoup de ce que le visiteur perçoit comme inhabituel et exotique. Le reste de l'article est également remarquable pour sa description de l'avant-garde artistique et intellectuelle haïtienne contemporaine. Au lieu d'imiter de nombreux auteurs qui décriaient les classes supérieures d'Haïti, les considérant comme une élite sur-éduquée singeant la France et n'étant pas authentiquement haïtienne, Porter admire leur lutte contre la répression à la fois interne et externe, soutenant qu'elles montraient le chemin dans la construction d'une image plus réaliste d'Haïti qui ne serait pas fondée sur un exotisme romantique ou sur le pittoresque.

Porter relatait son expérience afin de combattre les préjugés existant de longue date sur Haïti, mais ce faisant il a aussi rendu ses lecteurs conscients du grand nombre d'artistes et d'intellectuels haïtiens qui pouvaient parler 


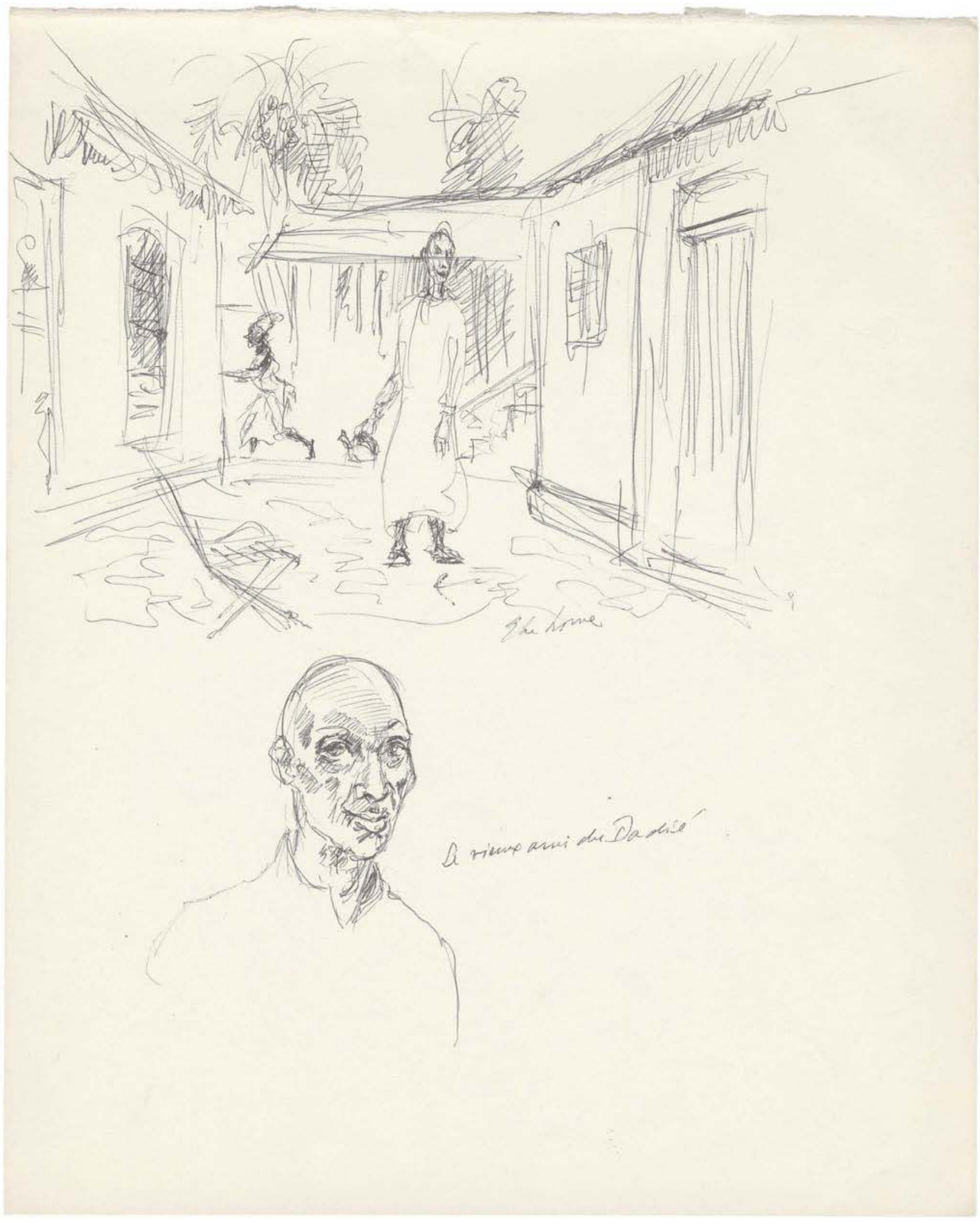




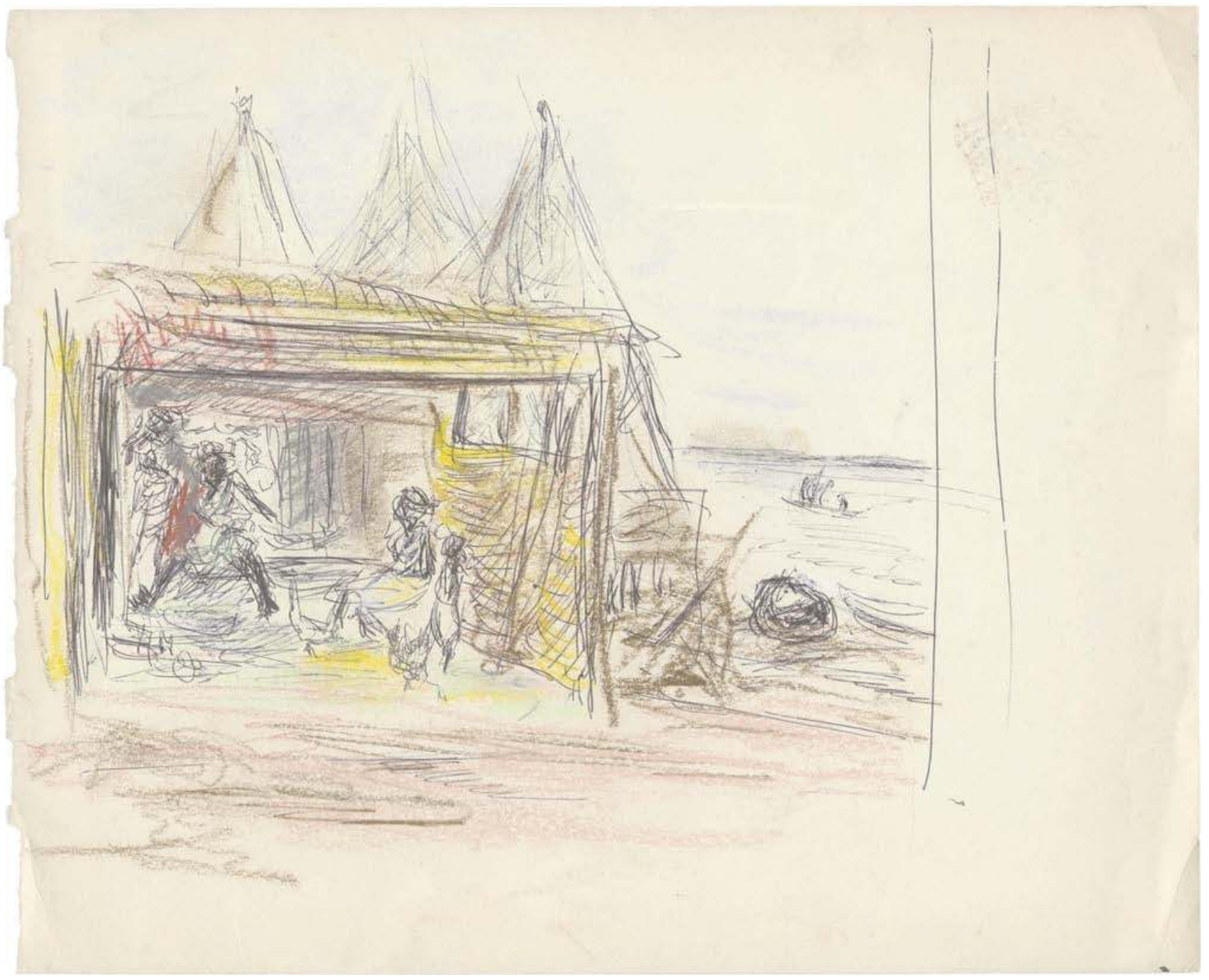

fig. 3

Croquis de James Amos Porter. James Amos Porter Papers, Robert W. Woodruff Library, Emory University, Atlanta, GA, Manuscript, Archives \& Rare Book Library. (C) James Amos Porter Estate. pour eux-mêmes. II les a guidés vers les voix indigènes qui avaient longtemps été ignorées aux États-Unis comme I'historien Dantès Bellegarde, le romancier-anthropologue-activiste Jacques Roumain ou les nombreux artistes haïtiens doués qui ont enfin été «découverts» par le monde extérieur à cette époque. II cite ensuite spécifiquement les noms de Pétion Savain (fig. 4), Charles [sic: Géo] Remponeau, Jean-Baptiste Bottex (le frère moins célèbre de Seymour Bottex), René Vincent, «Joseph Rigaud²» et A[ntoine] Derenoncourt. Et il ajoute «le brillant touche-à-tout Albert Mangonès, [qui] réussit à dominer les champs de l'architecture et de la sculpture». II conclut ensuite: "L'espoir d'Haïti repose sur ces jeunes nouveaux leaders qui ne font pas de quartier à l'exotisme baroque ou au néoromantisme timide avec son accent sur le faussement pittoresque. » (Ibid. : 210)

La liste de Porter inclut le peintre haïtien le plus important des années 1930, Savain, et plusieurs fondateurs du Centre d'art de Port-au-Prince tels que Mangonès et Remponeau, même s'il se trompe sur le prénom du second. Étant donné que ses recherches avaient pour but de produire une étude exhaustive sur les artistes les plus accomplis, les lecteurs familiers avec I'histoire de l'art haïtien remarqueront qu'il manque, dans cette seule publi- 
cation de ses travaux, plusieurs noms clés de cette première période comme Philomé Obin, Lucien Price et Luce Turnier. De plus, malgré son puissant plaidoyer en faveur de l'espace à donner aux artistes et aux intellectuels haïtiens, son long article ne comprend aucun de leurs énoncés artistiques ni aucune représentation de leurs œuvres bien que nous sachions qu'il a rencontré un grand nombre d'entre eux. (En outre, il illustre son article avec quatre de ses propres peintures produites en Haïti plutôt que d'inclure des œuvres haïtiennes.)

L'évaluation et l'analyse de l'art contemporain représentent toujours un défi, en particulier pour Porter qui est arrivé à Port-au-Prince aux premiers jours exaltants de la «renaissance» de l'art haïtien, alors en pleine explosion et se développant de façon exponentielle. Comme la plupart des chercheurs, il a produit ses évaluations les plus pertinentes quand il était soutenu par d'autres recherches. II a remarqué qu'il y avait sur l'art haïtien une «pénurie de publications » qu'il aurait pu rassembler, n'ayant récolté que treize éléments à comparer aux soixante-quatorze catalogues d'exposition, brochures, articles et ouvrages d'études sur l'art qu'il a collectés pendant qu'il était à Cuba (Porter 1946a). Sans surprise, son rapport final de vingt-sept pages au General Education Board contient plus d'analyses profondes sur l'art cubain que sur la peinture haïtienne, bien que son explication de l'architecture haïtienne soit perspicace et bien fondée, provenant probablement d'informations rassemblées au cours de ses entretiens avec Mangonès.

Pourtant, dans le même temps, le long rapport non publié de Porter offre un regard important et sans détours sur le «réseau d'actes et de paroles et d'écrits » d'un artiste-chercheur qui est allé en Haïti pour entamer des discussions diasporiques noires, pas seulement par des entretiens de recherche mais aussi par des représentations expressives (fig. 2 et 3). D'une certaine manière, nous pouvons dire que, pendant qu'il était en Haïti, les inclinations artistiques de Porter ont eu le dessus sur ses recherches. Car, comme il le remarque, «j'ai trouvé le peuple et le paysage haïtiens d'un intérêt si extraordinaire que j'étais là-bas capable de consacrer dix toiles à des sujets sans épuiser le moins du monde mon inspiration» (ibid.: 3). (En comparaison, il a produit seulement deux peintures pendant son séjour à Cuba, bien qu'il ait fait «de nombreux croquis au pastel»). II remarque que les artistes haïtiens se sont, en retour, grandement intéressés à son travail, discutant avec lui de ses sujets (des portraits de travailleurs haïtiens, "qu'ils considéraient à raison comme le "vrai peuple haïtien" "), de ses matériaux et de ses techniques (ibid.: 25).

Le rapport final de Porter au General Education Board souligne aussi ses discussions avec un certain nombre d'Haïtiens éminents, dont Price-Mars, Odette Rigaud (Bureau d'ethnologie), René Salomon, Stéphen Alexis (qui était alors conservateur du Musée national), et Remponeau et Price au Centre d'art. Par ailleurs, il note qu'il a fait de fréquentes visites au Centre d'art afin d'observer les progrès faits par les étudiants assistant aux cours de dessin et de peinture. Durant cette période, c'était le seul endroit à Port-au-Prince où l'on pouvait obtenir une formation avancée en art. Bien qu'il soit assez impressionné par la vision de DeWitt Peters,
2. II s'agit très probablement de Jeanne Élie-Joseph Rigaud, car Porter a déformé plusieurs des noms d'artistes qu'il cite, mais dans la mesure où nous ne pouvons en avoir la certitude, nous reprenons tels quels les noms et prénoms qu'il a notés. 
qui avait fondé le centre avec l'aide du gouvernement haïtien, Porter avait de profondes inquiétudes quant à sa politique éducative:

Néanmoins, ce serait une amélioration si M. Peters voulait bien abandonner cette pratique étroite de limiter l'expérience esthétique de l'artiste-étudiant à ce qu'il peut déjà connaître de crainte qu'il n'apprenne trop. Quels que soient les acquis de l'étudiant, que ce soit par l'entraînement ou l'expérience, il n'est pas encouragé par M. Peters à analyser le sujet qu'il rend ou les méthodes qu'il emploie dans son rendu. II s'agirait plutôt de faire de grands efforts pour aboutir à ce que $M$. Peters appelle la fraîcheur et l'originalité dans l'approche. La preuve de cette censure réside dans le fait que ceux qui sont réellement naïfs, parmi les élèves de $M$. Peters, peignent naïvement, tandis que ceux qui ne le sont pas peignent par imitation, par considération pour M. Peters. (Ibid. : 18-19)

Porter fait ensuite l'éloge de Remponeau, l'un des fondateurs du Centre d'art et l'un de ses plus influents professeurs, qu'il voit comme un important contrepoids à Peters:

Son expérience a consolidé les espoirs de ceux qui sont venus au Centre d'art pour une formation académique solide dans le dessin et la peinture. On peut sûrement dire que son influence va contrebalancer la tendance de la direction du Centre d'art à garder les yeux fixés sur la possibilité de ventes lucratives d'art primitif sur un marché américain qui a déjà surinvesti sur ce type de matériel. (Ibid.: 19)

Le désir de préserver la nature autodidacte des peintres naïfs, qui incarneraient et captureraient le mieux l'«authentique» essence haïtienne dans leur œuvre, n'a fait que s'accroître avec l'arrivée du poète et écrivain américain Selden Rodman, qui a codirigé le centre de 1949 à 1951 et dont les écrits ont dominé le champ des études d'art haïtien jusqu'aux années 1980. Porter est un commentateur précoce et perspicace des implications politiques et racistes de ce qui deviendra la dichotomie signifiante dans les jugements populaires sur l'art haïtien. Comme il l'a noté, «il ne peut y avoir aucun doute que l'attitude de M. Peters est une autre expression de cette condescendance que tant d'étrangers bien intentionnés adoptent envers un peuple dont on leur a dit qu'il était arriéré» (ibid.: 19). Le défi de Porter, qui perdurera dans les jugements sur l'art haïtien, était d'évaluer la qualité de styles artistiques variés, des artistes modernistes de formation académique, que Porter était plus à l'aise pour évaluer, jusqu'aux peintres naïfs et populaires. De plus, il voulait trouver une façon de débattre des extrémités de ce spectre comme de deux incarnations d'une expression authentiquement haïtienne qui parlerait à la fois aux racines africaines et aux réalités contemporaines du Nouveau Monde. II a finalement conclu qu'il fallait dépasser cette pensée binaire à propos de l'art haïtien (ibid. : 20).

Cette pensée binaire, cependant, continuera à être appliquée par des étrangers, influençant lourdement la réception de l'art haïtien dans les galeries, les musées, les organisations de financement et le marché de l'art. Les recherches de Porter sur les débuts de l'art haïtien et sa lutte 
contre cette pensée binaire ne se sont malheureusement pas concrétisées à l'époque où il l'espérait. Excepté dans son article pour Opportunity, il n'a jamais partagé ses idées sur ses études et les artistes haïtiens dans un écrit publié. À sa mort en 1970, il travaillait encore sur son livre The Black Artist, qui aurait intégré ses réflexions sur les influences artistiques africaines dans le monde occidental et ses vastes recherches à Cuba et en Haïti, ainsi que celles qu'il a menées par la suite au Brésil (Driskell 1992: XXXIII). Néanmoins, il a rapporté de ce voyage d'étude et de peinture assez de notes et de diapositives pour ouvrir un nouveau cours sur l'art latino-américain à la Howard University (Davis 1985: 90). Ses conférences et le matériel qu'il a rendu accessible à ses étudiants ont eu une influence inattendue sur les futures générations d'artistes et de chercheurs qui ont poursuivi ses efforts pour que des dialogues diasporiques puissent célébrer les travaux d'artistes noirs et combattre le racisme grâce à un front unifié par la compréhension interculturelle. Ses notes et ses photographies survivent et constituent un trésor pour une évaluation plus approfondie de l'histoire de l'art haïtien durant la période cruciale du milieu du siècle dernier. Après le décès de sa fille Constance Porter Uzelas en 2012, ses archives ont été vendues aux enchères et sont désormais hébergées par l'Emory University d'Atlanta où, bien qu'elles ne soient pas encore traitées, elles seront accessibles aux chercheurs.

\section{Loïs Mailou Jones Pierre-Noël, peintre et professeur}

Le flambeau du dialogue diasporique noir a été pleinement repris par Loïs Mailou Jones Pierre-Noël (1905-1998), collègue de longue date de Porter à la Howard University. Bien qu'elle n'ait pas suivi de formation spécifique en histoire de l'art, et se sentant peut-être éclipsée par Porter, Jones Pierre-Noël a également mené des recherches intensives sur l'art de la diaspora noire grâce à des financements de la Howard University, partant d'abord en Haïti, puis en Afrique de l'Ouest. Comparée à Porter, cependant, elle resta liée avec Haïti toute sa vie de façon très intime, ce qui a profondément inspiré son travail d'artiste, ses recherches et sa pratique curatoriale. Elle a visité Haïti pour la première fois à l'été 1954, invitée par le gouvernement haïtien. Il s'agissait aussi de son voyage de noces car elle avait épousé l'année précédente l'artiste et graphiste haïtien Louis Vergniaud Pierre-Noël (1910-1982). Pendant cette première visite, elle produisit une quarantaine d'huiles et d'aquarelles. Elle exposa une sélection de ces œuvres au Centre d'art en septembre.

Ce premier été, Jones Pierre-Noël enseigna aussi au Centre d'art, pendant les congés de Peters, et également au Foyer des arts plastiques fondé en 1950 par des dissidents du Centre d'art. De tels postes d'enseignement étaient devenus des arrangements standard, en particulier au Centre d'art, et de nombreux artistes américains enseignaient là-bas, souvent en échange d'une place d'atelier. Jones a trouvé le travail et les artistes à ces deux endroits "chaleureux et inspirants". Elle a écrit avoir rencontré Rigaud Benoît, Castera Bazile, Robert Saint-Brice, Jasmin Joseph, Murat Brierre, Montas Merisier, Dieudonné Cédor, Wilson Jolicoeur, Joseph Jacob, Raymond Oliveri, André Dimanche et les frères Maurice. Également «au nombre de [ses] amis » les sculpteurs René Exume, Luckner Lazard, «et bien d'autres». Elle note aussi avoir été particulièrement impressionnée par le travail 


\section{ci-contre}

fig. 4

Petion Savain, Scène de marché, huile sur toile,

1961. Courtesy Chicago

Gallery of Haitian Art.
d'Antonio Joseph et Nehemy Jean, ajoutant qu'il reflétait leurs expériences à l'étranger. En bref, elle a été marquée par l'abondance de techniques et de styles personnels qu'elle pouvait voir dans les travaux de ses nouveaux amis (Jones Pierre-Noël s.d. : 1-2).

Enseigner en Haïti, cependant, s'est révélé une nouvelle aventure pour laquelle Jones Pierre-Noël a dû s'adapter. Elle avait suivi une formation artistique significative, commençant pendant son adolescence par les cours de la School of the Museum of Fine Arts à Boston, où elle a poursuivi ses études après la fin de ses études secondaires. Elle a ensuite obtenu un certificat de la Boston Normal Art School (désormais le Massachusetts College of Art), et elle a aussi étudié à la Designers Art School de Boston, à la Columbia University (où elle a rencontré pour la première fois Pierre-Noël en 1934) et à l'Académie Julian à Paris (grâce à une bourse du General Education Board en 1937). Sa formation très classique l'a aidée à démontrer qu'une artiste femme et noire était tout à fait capable de parvenir aux plus hautes sphères des beaux-arts, du naturalisme académique aux styles cubiste et impressionniste. Et elle a apporté sa vaste formation classique dans sa salle de classe de la Howard University, où ses étudiants apprenaient les éléments et principes du dessin, la théorie des couleurs et le modèle vivant.

Cependant, cet été-là en Haïti, en réfléchissant à son enseignement, Jones Pierre-Noël a écrit: « Il m'est bientôt apparu que la plupart des artistes du Centre d'art ne s'intéressaient pas à l'apprentissage des méthodes académiques impliquant la perspective, les théories des couleurs ou les techniques modernes. Ils préféraient travailler dans un style plus naïf. " Tout le monde ne considérait pas cela comme une bonne chose, comme l'a noté plus haut Porter dans ses observations au centre durant l'hiver 1945. Arrivant dix ans plus tard, Jones Pierre-Noël était elle aussi clairement mal à l'aise avec cette idée que l'authentique art haïtien ne pouvait être que celui des «primitifs» stylistiques qui avaient capté l'attention du monde de l'art. Heureusement, elle a aussi noté qu'elle avait trouvé de nombreux étudiants intéressés par son approche plus académique: "La meilleure réaction que j'ai eue venait d'artistes plus jeunes - entre 16 et 20 ans - qui étaient désireux de recevoir une formation de niveau professionnel. Les cours de modèle vivant et d'aquarelle avaient lieu dans la cour du Centre d'art. Ils rassemblaient entre vingt et vingt-cinq étudiants. " Elle donnait des cours particuliers dans son atelier de Bourdon, un quartier de Port-au-Prince, à quelques étudiants «au talent exceptionnel» dont Michèle Manuel, Monique Villedrouin, ClaireEdith Mark et Louis Fombrun. Elle les emmenait également à des excursions de peinture "de plein air», au marché de Kenscoff et au Cap-Haïtien, pour peindre la Citadelle et les paysages locaux (ibid.).

Pour Jones Pierre-Noël, mettre ainsi l'accent sur l'art académique et les grandes traditions occidentales des avant-gardes de l'art moderne était une offensive créative qui défendait sa position dans le monde de l'art et proclamait ce dont une artiste femme issue d'une minorité était capable. Jusqu'à ce moment, sa carrière, avec quelques notables exceptions, montrait sa maîtrise des traditions picturales européennes: le naturalisme académique, le traitement méticuleux de la peinture, mais aussi les réactions «de plein air » à la couleur et à l'atmosphère de l'école impressionniste, avec 


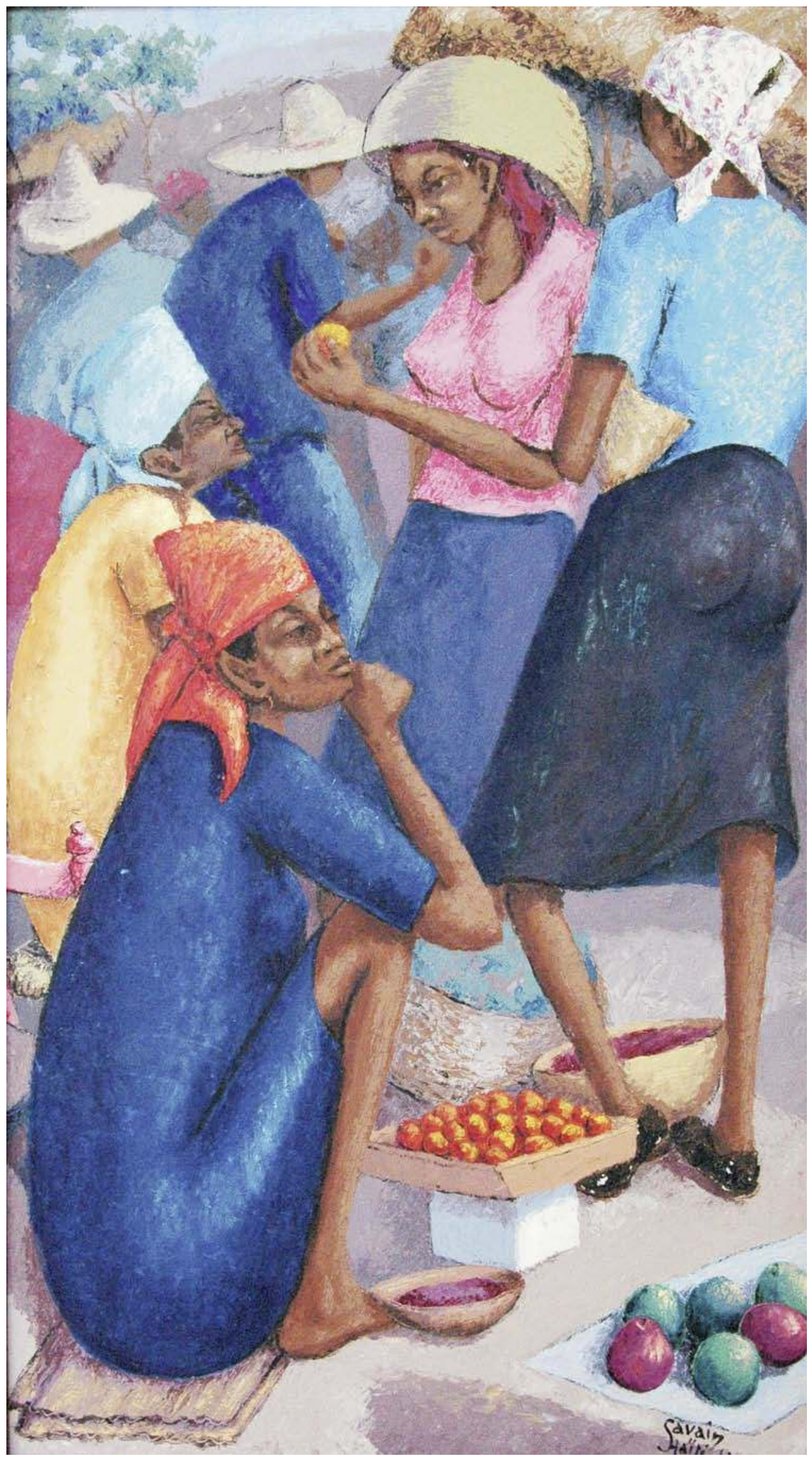


fig. 5

Michele Manuel, Cap

Haïtien, acrylique, 1973.

Photo Art, Architecture

\& Engineering Library,

University of Michigan

Library. Courtesy Galerie

Expressions. (C) Michele

Manuel.

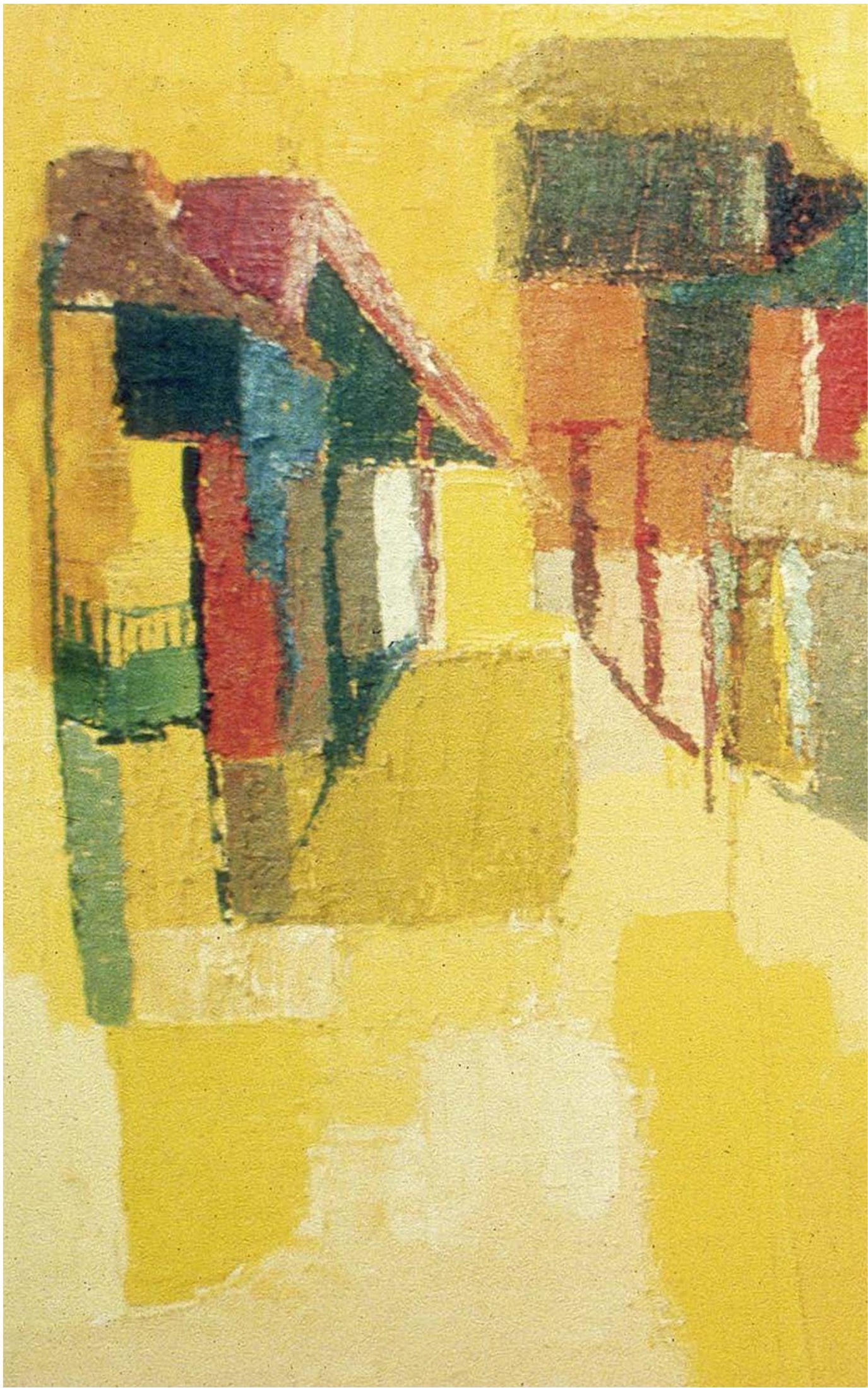




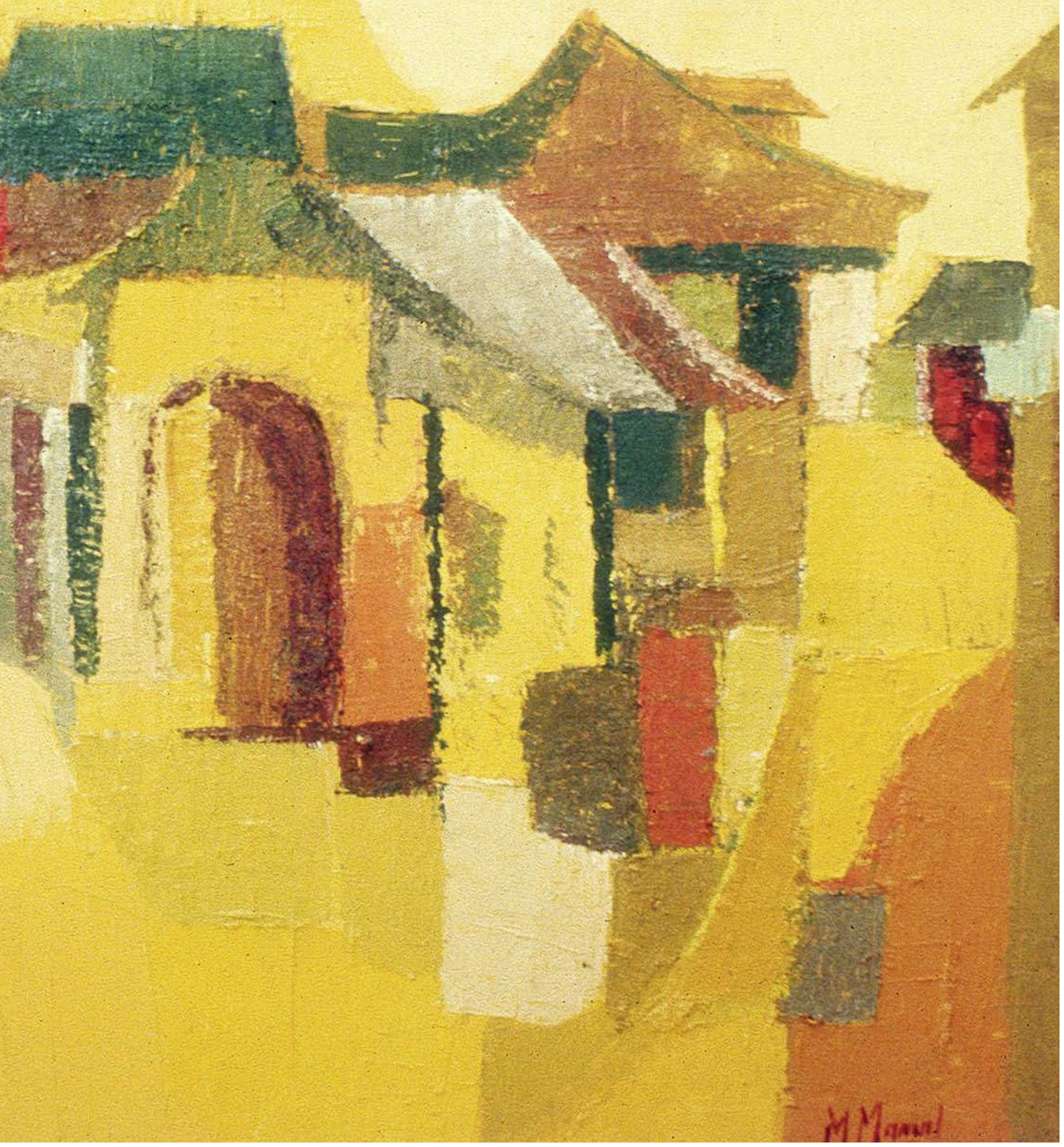


quelques incursions dans le cubisme. Voilà le style et les intérêts qu'elle a emmenés avec elle en Haîti en 1954. Mais, après ce premier contact avec Haïti, son style personnel a subi un changement radical. D'une certaine manière, elle réagissait à son époque: les œuvres naturalistes de style académique et les styles impressionniste et postimpressionniste devenaient obsolètes face à l'expressionnisme abstrait et aux réponses variées du monde de l'art à la décomposition de mouvements monolithiques - du pop art à l'op art, la performance artistique, etc. Le monde de l'art était en pleine expansion, et les collectionneurs recherchaient des artistes et des styles hors de New York, Paris ou Berlin. Les artistes haïtiens autodidactes de style populaire avaient déjà atteint la célébrité internationale avant la fin des années 1940.

Haïti a changé Jones Pierre-Noël bien avant qu'elle ne change Haïti. Avant la fin des années 1950, elle a rejeté son style académique original et s'est mise à utiliser des formes stylistiques et des sujets haïtiens comme inspiration pour l'abstraction. À ce moment-là, elle avait un atelier en Haïti où elle vivait et travaillait pendant les congés universitaires de la Howard University. Cela a permis à son travail de dialoguer d'encore plus près avec l'art haïtien, dans lequel elle trouvait des affinités avec son propre mélange de représentations naturalistes et d'abstraction caractéristique des styles des artistes naîfs comme des plus sophistiqués.

\section{Jones Pierre-Noël, conservatrice et historienne de l'art}

Jones Pierre-Noël a aussi canalisé son énergie professionnelle dans la recherche en histoire de l'art, dans des projets d'expositions mettant en valeur les accomplissements d'artistes noirs et dans la construction en profondeur de dialogues diasporiques noirs (fig. 6). Elle a constamment défendu l'idée de la haute qualité artistique à laquelle pouvaient parvenir les Noirs et les femmes grâce à une diversité de styles qui parlait à leur communauté et témoignait également d'un fort engagement dans la modernité artistique. Comme elle l'écrit, après avoir témoigné du grand talent et des réussites des artistes en Haïti depuis les années 1950, «j'ai trouvé d'une importance primordiale la promotion de l'évolution de l'art haïtien à l'étranger» (ibid.: 2). L'art haïtien contemporain est devenu un intérêt particulier de Jones Pierre-Noël aussi bien en termes de recherche que d'organisation d'expositions. La première eut lieu en 1965 et rassemblait les travaux de Jean-Claude Garoute (Tiga) et de seize autres céramistes haïtiens, dont Patrick Vilaire, Yolande Étienne, Wilfrid Austin (Frido) et Hilda Williams (fig. 9). Cette vaste exposition comprenait près de quatre cents travaux de céramique et a été présentée parallèlement à trente-deux huiles et aquarelles d'Haïti réalisées par Jones Pierre-Noël. Elle en était la principale organisatrice, tandis que Porter a fourni l'introduction à l'exposition, dans laquelle il attirait l'attention du visiteur sur la présence de la religion haïtienne dans les œuvres, "dans des motifs décoratifs de signification symbolique (vèvè et certaines formes animales)", et faisait l'éloge du "vaste étalage» de figurines dépeignant "la vie quotidienne en Haïti dans toutes ses variétés de couleurs et de mouvements». "Fondé sur la technique de l'engobe impliquant l'utilisation d'argiles naturellement colorées, poursuivait Porter dans son explication, c'est un art à la fois de vérité et de sophistication. » (Porter 1965) 
Autant pour Porter que pour Jones Pierre-Noël, la vérité et la sophistication étaient deux concepts moteurs dans leur approche de l'art haïtien. Mais ces idées devaient être maniées avec prudence et dextérité. La notion de vérité dans l'œuvre d'art haïtienne devint un code pour mesurer son authenticité, c'est-à-dire à quel point elle reflétait et incarnait un puits profond de racines culturelles et raciales. Comme l'ont fait remarquer de nombreux auteurs et collectionneurs, en raison de la grande popularité des œuvres d'art naïf haïtiennes sur les marchés locaux de l'art, particulièrement touristiques, un ensemble de stéréotypes racistes et primitivistes en sont venus à représenter ce qui était considéré comme stylistiquement haïtien. Toute œuvre ne comprenant pas certains sujets et marqueurs stylistiques - comme des couleurs symboliques éclatantes, une utilisation restreinte de la tridimensionnalité, un usage de la perspective intuitive au lieu de la perspective linéaire, voire le rejet de l'ensemble des techniques de perspective, par exemple - risquait d'être considérée comme trop sophistiquée et donc non authentiquement haïtienne. Cela rendait bien sûr impossible à la fois d'établir un discours sur ce qui constituait la qualité de l'art haïtien et, pour l'artiste issu d'une minorité, d'obtenir du succès dans le monde de l'art.

Porter et Jones Pierre-Noël ont dû lutter contre cela dans leur production artistique tout au long de leur vie. En tant qu'artistes d'origine africaine, ils devaient prouver qu'ils maîtrisaient les techniques académiques propres au monde de l'art occidental. Pour assurer qu'ils n'étaient pas vieux jeu, cependant, ils devaient aussi montrer qu'ils pouvaient choisir de rejeter ces standards et appliquer dans leurs œuvres l'abstraction et les théories conceptuelles. Les peintres du début du xx $x^{e}$ siècle comme Pablo Picasso l'avaient fait en se tournant vers l'art africain. Mais les artistes d'origine africaine devaient démontrer que leurs explorations étaient un choix intellectuel dans un but expressif et non simplement un manque d'originalité, de niveau ou de pratique, voire pire, une affinité raciale innée.

Porter et Jones Pierre-Noël ont transféré ce paradoxe dans leurs études sur l'art haïtien et ni l'un ni l'autre n'est parvenu à une conclusion entièrement satisfaisante. Nous avons une meilleure vision des négociations de Jones Pierre-Noël pour parvenir à un équilibre car ses recherches ont abouti non seulement à faire avancer ses propres pratiques artistiques, mais aussi à de nombreux projets d'expositions, des conférences et des publications. II faut rappeler qu'elle avait l'avantage de l'intimité et du temps: l'intimité, puisque suite à son mariage avec Vergniaud Pierre-Noël elle vivait en Haïti pendant une bonne partie de l'année; le temps, car dans les années 1960 et 1970 ses études pouvaient s'appuyer sur des décennies supplémentaires de développement et de reconnaissance de l'art haïtien. Elles pouvaient également être renforcées par le Black Arts Movement, en pleine expansion aux États-Unis et, partout dans le monde, par des collaborations et des célébrations de la diaspora noire proclamant fièrement les succès des Noirs et leur égalité avec les Blancs. Des financements de recherche supplémentaires de la Howard University lui ont aussi permis de voyager en Afrique, où elle a pu établir des comparaisons de première main avec l'art africain ancien et contemporain et les relater dans ses dialogues avec Haïti. 
3. Nous reprenons ici tels quels les noms et/ou prénoms cités par Porter en mentionnant entre crochets l'hypothèse la plus probable concernant l'artiste auquel ils se rapportent.
À l'été 1968, la Howard University a attribué à Jones Pierre-Noël des financements de recherche pour étudier des artistes haïtiens contemporains et documenter leurs travaux par des diapositives. D'après le rapport initial, il est clair qu'elle considérait cette «aventure des plus stimulantes» sous l'angle du dialogue diasporique noir. Elle a écrit que ce projet de recherche était «grandement motivé par l'accroissement actuel de l'intérêt pour "l'art des Noirs" ". De plus, bien que cet été de recherche se soit concentré uniquement sur «l'art en Haïti », elle le voyait, d'après le titre de son premier rapport, comme une première marche dans ce qu'elle envisageait déjà comme une étude académique plus large sur les «arts visuels noirs » (Jones Pierre-Noël s.d. [1968]). Cet été-là, elle a enquêté auprès des principaux centres d'art, des galeries, des ateliers et des collections privées, et a même expertisé les fresques murales de l'aéroport. Elle rapporte qu'elle s'est entretenue avec plus de trente artistes et a collecté des données biographiques documentaires.

Dans le premier rapport de Jones Pierre-Noël à la Howard University, elle plaide pour l'importance de son étude en expliquant qu'Haïti est l'un des centres artistiques les plus productifs des Caraïbes. Elle relate ensuite I'histoire désormais bien connue de l'arrivée de Peters, de la création du Centre d'art et de la «floraison» de la peinture haïtienne. Comme Porter avant elle, elle écrit: «C'était [l'opinion de Peters] que l'artiste haïtien devait développer son propre style au sein d'un cadre primitif, préservant ainsi sa liberté et l'originalité de son expression. » Elle cite ensuite les noms d'Obin, Hector Hyppolite, Bazile, Wilson Bigaud, Benoît, Jacques-Enguerrand Gourgue, Fernand Pierre et d'autres «dont j'ai également photographié les œuvres». Elle passe rapidement cependant à l'existence d'autres groupes artistiques qui produisent des œuvres en dehors de «l'influence dominante du "primitivisme" » qui a «entravé la progression de leurs œuvres». Elle décrit la formation du Foyer des arts plastiques, qu'elle appelle le «deuxième mouvement artistique » d'Haïti, notant les noms de Lazard, Max Pinchinat, Price, [Roland] Dorcely, Exume, [Dieudonné] Cédor, Maurice Borno, Spencer Despas, Turnier, Gesner Armand, Antonio Joseph et Jacques Gabriel. Elle identifie ensuite un troisième mouvement artistique en Haïti, la nouvelle école d'art moderne, avec les artistes Garoute (Tiga), Vilaire et Austin (Frido). Le rapport complet, qui compte sept pages, se poursuit par une liste complémentaire incluant des «artistes qui méritent d'être surveillés» (dans laquelle on trouve Jolicoeur [Emmanuel ou Wilson?], René Exume, Elzire Malebranche, [Laurent?] Casimir, Calixte Henri, Joseph Jacob, Raymond Olivier, Joseph Raymond, Pierre Edugene et Florence Martinez) et une liste de sculpteurs (Georges Liautaud, André Dimanche, Murat Brierre, Hilaire Mollenthiel, Ludovic Booz, André Lafontant, Jean Baptiste [Pericles?] et les frères Maurice ${ }^{3}$ ) [ibid. : 2-3].

Il semble que le but principal de Jones Pierre-Noël, dans ce premier essai de chronique de l'histoire de l'art haïtien, ait été de submerger ses lecteurs par la simple quantité d'artistes engagés et talentueux. De plus, plutôt que de présenter ces derniers comme divisés en groupes concurrents, elle insiste sur le fait que «ces trois mouvements contribuent tous à une riche croissance de l'art en Haïti ». En insistant sur le grand nombre d'artistes qui travaillent en dehors du style catalogué «populaire», «primitif» et «naïf», 


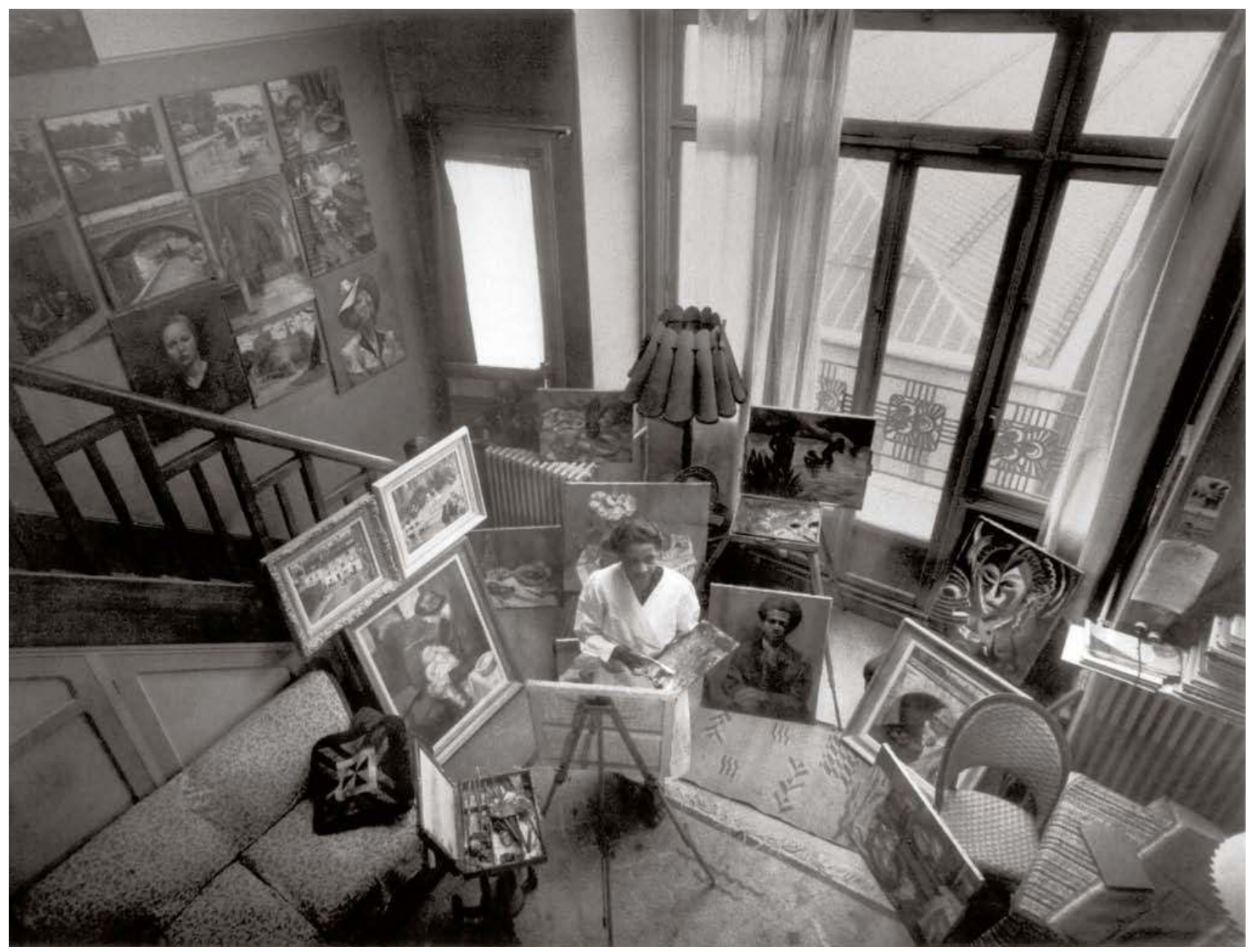

fig. 6

Loïs Mailou Jones Pierre-

Noël dans son atelier à

Paris, 1938. Loïs Mailou

Jones Papers, Moorland

Spingarn Research

Center. Howard University,

Washington, DC. () Loïs

Mailou Jones Trust. 
LERAMEBADFGAIS

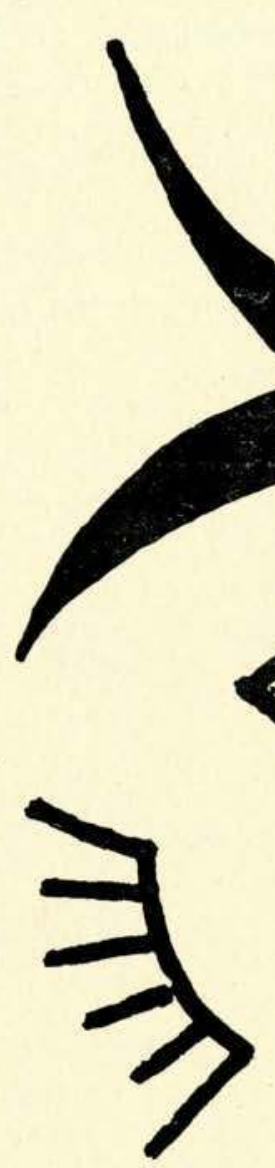

EXPOSE 
ainsi qu'en mettant en valeur trois mouvements et non juste une opposition binaire entre «primitifs" et "sophistiqués», elle cherche à mettre l'accent sur la diversité des styles, des formations et des objectifs artistiques qu'elle avait trouvée dans la culture vivante de l'art contemporain en Haïti. Dans les mouvements qu'elle a identifiés, elle insiste sur le sérieux des intentions et le mérite des travaux des artistes haïtiens d'avant-garde, qui «ont évité d'être pris dans la vacuité des œuvres commissionnées pour approvisionner les achats touristiques » (ibid. : 2-4).

Au cours de cet été, Jones Pierre-Noël a réalisé plus de trois cent soixante diapositives couleur et a fièrement relaté: "La Howard University peut affirmer qu'elle a la documentation la plus vaste et la plus actualisée sur l'art en Haïti, sous la forme de diapositives et de données biographiques. ” (Ibid.: 1) Dans les années suivantes, elle a continué à enrichir cette collection avec ses voyages en Haïti et en Afrique, portant cette iconothèque à plus de mille diapositives ayant intégré les archives de la Howard University. Elle a aussi poursuivi un programme curatorial actif pour promouvoir les artistes haïtiens. Par exemple, elle a organisé en 1969 la première exposition personnelle de Vilaire à la Pan American Union de Washington.

Jones Pierre-Noël a intitulé ce premier projet d'étude «Les arts visuels noirs", comprenant que ses recherches et sa documentation d'artistes en Haïti n'étaient qu'un premier pas dans la production d'une étude plus globale des arts contemporains de la diaspora noire. En 1970-1971, grâce à une nouvelle aide financière de la Howard University, elle a conduit des entretiens et rassemblé des photographies et du matériel sur les arts contemporains de quatorze pays africains. Insatisfaite de sa première série de diapositives et d'entretiens en Haïti, elle a rapidement identifié un domaine qui avait besoin d'une documentation et d'une promotion plus approfondies: l'art des femmes haïtiennes. Sa troisième bourse de recherche de la Howard University est arrivée en 1973, spécifiquement pour l'étude des femmes artistes caribéennes et afro-américaines (fig. 8).

Le résultat immédiat de l'étude de Jones Pierre-Noël a été une exposition de femmes artistes qu'elle a organisée pour la galerie Acts of Art à New York et qui s'est tenue du 25 juin au 13 juillet 1974. En plus d'une artiste du Guyana, sept de Jamaïque et dix afro-américaines, elle a présenté treize artistes haïtiennes: Tamara Baussan, Rose-Marie Desruisseau, Marie-José (Nadal-)Gardère, Édith Hollant, Gizou Lamothe, Elzire Malebranche, Michèle Manuel, Claude Maximilien, Gilda Thebaud Nassief, Andrée Georges Naudé, Micheline Prézeau, Maud Gerdes Robart et Luce Turnier. Ses recherches sur les femmes artistes ont aussi été présentées en bonne place au Diaspora 2: deuxième Festival de la diaspora africaine du Nouveau Monde, qui a eu lieu à Port-au-Prince en août 1979. Elle a été une intervenante importante à l'Institut haïtiano-américain, présentant la conférence «Femmes artistes noires».

Dans son introduction à l'exposition, les rapports qui ont suivi et les conférences sur ses études, Jones Pierre-Noël énonce clairement ses objectifs. Avant tout, elle veut remédier à l'exclusion des femmes artistes des histoires de l'art standard et, en même temps, célébrer la riche diversité

\section{ci-contre}

fig. 7

Loïs Mailou Jones

Pierre-Noël, brochure de l'exposition

"Ceramebadagais",

Howard University,

avril 1965. Loïs Mailou

Jones Papers, Moorland

Spingarn Research

Center. Howard University,

Washington, DC. (C) Loïs

Mailou Jones Trust. 
des sujets et des styles dans la production artistique de femmes accomplies et de grand talent. De telles études et proclamations étaient de plus en plus communes dans les années 1970, avec la montée de mouvements artistiques féministes. Le projet de Jones Pierre-Noël est également important pour la mise en avant d'un dialogue entre artistes femmes issues des minorités et caribéennes, qu'elle appelait «femmes du tiers-monde partout». Car, comme elle le note, «dans un monde d'hommes, toutes les femmes sont des femmes du tiers-monde». En produisant son rapport de recherche constitué de biographies d'artistes femmes caribéennes et afroaméricaines, elle soutient que «les femmes doivent travailler ensemble pour trouver leur propre structure et leur propre imagerie». Elle ne recherche cependant pas un style monolithique ou des sujets ouvertement féminins. Son étude met en valeur aussi bien des femmes travaillant dans les modes naturalistes traditionnels que d'autres dans des modes intuitifs et autodidactes; aussi bien celles qui utilisent l'art à des fins politiques (des artistes qui «luttaient avec leurs pinceaux») que les modernes qui adoptent l'abstraction. Elle souhaite une nouvelle évaluation fondée sur le dialogue direct qui serait au bénéfice mutuel de tous face aux réalités discriminatoires du monde de l'art, quels que soient le style ou le contenu. Avant tout, elle met au défi les artistes issus de minorités et caribéens de prendre en charge l'organisation de leurs expositions et de s'entraider, affirmant une «nouvelle unité" pour établir l'importance de la femme artiste (Jones Pierre-Noël 1973-1974: 1).

Comme Porter, Jones Pierre-Noël avait prévu d'utiliser ses recherches dans un livre « définitif » sur l'art haïtien contemporain. Elle n'a pas atteint cet objectif, peut-être en partie par défaut de maison d'édition, et clairement par manque de temps. (Comme Porter, elle a assuré une grosse charge de travail d'atelier et d'enseignement jusqu'à sa retraite en 1977, et elle a activement continué ensuite à produire et exposer son art.) En plus de ses rapports de recherche pour la Howard University, elle a écrit plusieurs articles pour diverses publications, dont le Haitian Art Newsletter (ÉtatsUnis), Le Nouveau Monde (Haïti) et National (États-Unis), et plusieurs textes et comptes rendus d'expositions. Ses écrits, de multiples façons, reprennent le style de son collègue Porter en mettant l'accent sur l'ampleur du champ de recherche pour montrer la réussite collective d'un groupe minoritaire, tout en documentant également les biographies, les formations et les réalisations des individus listés. Et, comme Porter, elle ne s'est pas appesantie sur le contexte social ou les descriptions et interprétations ekphrasistiques, hormis le fait d'expliquer le sujet (en particulier si c'est une représentation du vodou) ou de préciser si l'artiste emploie l'abstraction. Cela était peut-être dû à la brièveté des textes qu'elle produisait habituellement, et aussi au fait qu'en tant qu'artiste elle supposait que l'art devait parler pour lui-même à l'observateur. Mais cette rigueur formelle était le point de départ nécessaire pour accéder à la langue de prestige du monde de l'art. Elle proclamait que la biographie et l'œuvre d'un «artiste du tiersmonde", d'une femme et/ou d'un(e) Noir(e) étaient dignes de documentation au même titre que celles d'un homme «d'un pays développé».

L'ambitieux projet de recherche de Jones Pierre-Noël et ses écrits fondamentaux méritent d'être connus et considérés étant donné la large 
fig. 8

Loïs Mailou Jones

Pierre-Noël, brochure de

l'exposition «La galerie

d'art de l'Université de

Howard présente les

œuvres de Rose-Marie

Desruisseau, peintre

d'Haïti ", 16 Octobre-6

Novembre, 1974. Loïs

Mailou Jones Papers,

Moorland Spingarn

Research Center. Howard

University, Washington, DC.

(C) Loïs Mailou Jones Trust.

\section{HOWARD UNIVERSITY GALLERY OF ART \\ PRESENTS PAINTINGS BY}

\section{ROSE-MARIE

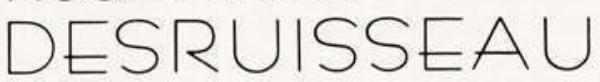 \\ OF HAITI}

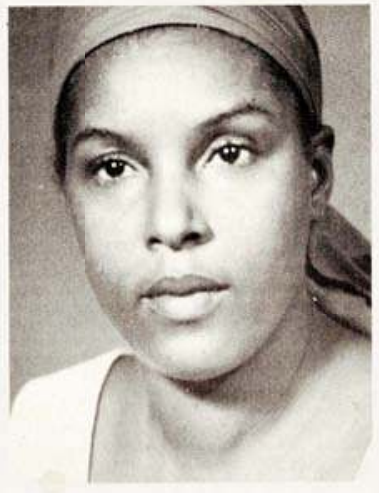

EXHIBITION

OCTOBER 16-NOVEMBER 6, 1974

\section{PORT-AU-PRINCE}

AND

PAINTINGS of HAIII

by

LOIS M. JONES

APRIL 9-MAY 6, 1965
Ceramics

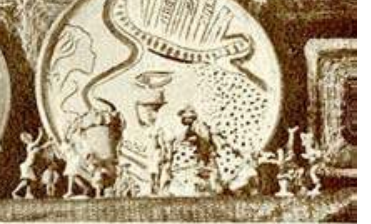

from Centre de Ceramique

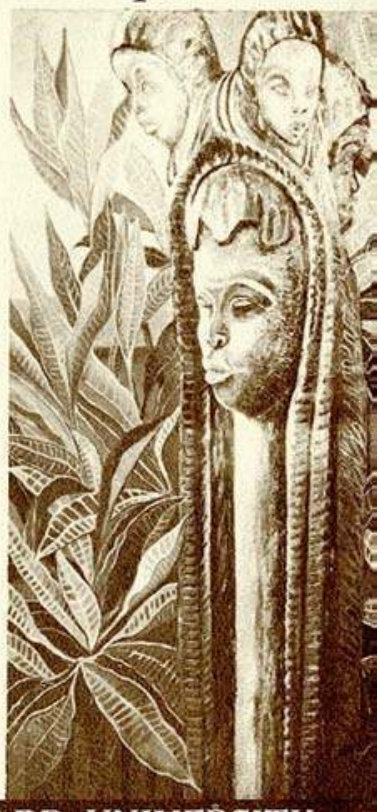

GALLERY OF ART, HOWARD UNIVERSITY

\section{fig. 9}

Affiche de l'exposition

"Céramiques haïtiennes

du Centre de Céramique,

Port-au-Prince et peintures

d'Haïti de Loïs M. Jones",

galerie d'art de l'Université

de Howard, 9 avril-6 mai 1965.

Loïs Mailou Jones Papers,

Moorland Spingarn

Research Center. Howard

University, Washington, DC

(c) Loïs Mailou Jones Trust. 
circulation et la domination des textes défendant presque exclusivement l'art haïtien de style populaire, à commencer par ceux de Rodman. Ses études élargissent le canon haïtien tout en démystifiant également les artistes, par exemple en discutant de leur formation et de la difficulté du travail investi dans leurs créations. Ses écrits, ses conférences, ses notes et ses archives, comme ceux de Porter avant elle, forment un contre-récit considérable aux écrits sur l'art haïtiens caractéristiques de l'époque.

Bien que Jones Pierre-Noël n'ait pas été capable de publier le livre « définitif » sur «l'art d'Haïti » qu'elle espérait, ses recherches ont circulé bien au-delà des salles de cours de la Howard University. Par une intéressante ironie de l'Histoire, la Budek Slide Corporation (désormais Media for the Arts) de Newport (Rhode Island) a publié en 1984 une brochure et des diapositives de son étude sur les femmes caribéennes et afro-américaines. Cette société a diffusé ses images et ses biographies d'artistes auprès de musées, de facultés et d'universités, rendant l'information disponible et prête à être intégrée à des conférences et des programmes de cours (Jones Pierre-Noël s.d.: 3). Et ces visuels (fig. 5) sont toujours accessibles via d'importantes banques de données comme la University of Michigan Art, Architecture and Engineering Library et ArtStor.org, bien qu'ils ne soient plus liés aux biographies de Jones Pierre-Noël et que les photographies ne soient plus créditées à son nom. Ses images de recherche circulent désormais dans une large matrice où elles peuvent être utilisées, analysées et comparées et déclencher avec un peu de chance les nouvelles conversations que cette artiste-chercheuse souhaitait voir naître.

\section{Conclusion}

La première fondation de l'histoire de l'art haïtien a été conçue sur des habitudes intellectuelles particulières, conduites et cultivées par des individus dont les goûts et intérêts personnels ont dicté les questions posées en dialogue avec des artistes haïtiens et leurs œuvres. Nous faisons bien de nous rappeler qu'aucune recherche n'est neutre, et que les canons des histoires de l'art se développent à partir de récits et de conversations localisés intégrant le désordre de circonstances personnelles. Ce n'est pas moins vrai d'un auteur comme Rodman, dont les comptes rendus exubérants mais primitivistes ont dominé le domaine pendant des décennies, que d'artistes-chercheurs afro-américains comme Porter et Jones Pierre-Noël. Ces derniers étaient clairement mal à l'aise avec le fait de privilégier une définition stylistique étroite d'un art haïtien réussi. Tous deux défendaient plutôt une plus grande diversité de styles et une sélection plus large d'artistes, et privilégiaient plus spécifiquement les «modernes" et les "sophistiqués" en tant qu'avant-garde légitime elle aussi authentiquement haïtienne. Cela était clairement relié à des préférences personnelles, en cela qu'ils étaient soutenus par une formation artistique académique classique approfondie ainsi que par l'étude des styles et designs modernistes européens. Mais en tant qu'éminents chercheurs afro-américains qui se trouvaient également être des artistes, Porter et Jones Pierre-Noël en sont venus à l'art haïtien par la fondation des principes du mouvement afro-américain New Negro; avant tout, ils estimaient que leur art et leurs recherches avaient besoin d'établir le talent des artistes noirs, la haute qualité de leurs travaux et leur aptitude à communiquer dans les domaines de l'avant-garde moderniste de 
manière aussi sophistiquée que les artistes hommes, européens ou euroaméricains blancs. Plus qu'étendre le canon, de telles recherches étaient un moyen de lutter ouvertement contre les stéréotypes raciaux omniprésents de cette époque, qui soulignaient l'infériorité noire et limitaient les droits des populations d'origine africaine.

Les écrits des deux chercheurs - rapports de recherche, conférences, brouillons et publications - ont en eux une qualité formelle stricte. Ils mettent l'accent sur les données biographiques, avant tout la formation, les participations à des expositions et les prix obtenus, plutôt que d'offrir des descriptions mimétiques et des passages ekphrastiques qui auraient conduit à se concentrer sur les interprétations d'œuvres d'art spécifiques. De bien des façons, une telle écriture de style journalistique semble être une réaction ouverte contre les comptes rendus sur l'art haïtien qui dominaient les années 1940 à 1970, et qui décrivaient celui-ci en des termes excessivement alourdis par l'émotion, le mysticisme et le primitivisme. Les textes de Porter et de Jones Pierre-Noël, quant à eux, relataient rigoureusement les origines, la formation, les expositions et les prix: l'importante langue de prestige du monde de l'art était récupérée et appliquée à un large groupe d'artistes noirs partageant une origine nationale. Une analyse en profondeur d'artistes et d'œuvres spécifiques est cependant perdue dans cette approche, et la lecture de l'histoire des débuts de l'art haïtien en est rendue frustrante pour les chercheurs et les connaisseurs à la recherche d'une dimension académique plutôt que d'un large relevé.

Ce qui est en jeu ici, c'est la façon dont les artistes viennent à être connus, et comment cette renommée proscrit typiquement (et limite) leur niveau de reconnaissance et de prestige au sein du monde des arts. Bien sûr, les œuvres peuvent parler pour elles-mêmes en cela qu'elles communiquent et opèrent dans un domaine esthétique de formalisme et de facture. Mais l'art génère des significations à travers de nombreuses couches complexes. De plus, au sein du monde de l'art et de ses marchés, l'idée de qualité innée a toujours grandement dépendu de la stature et de la biographie de l'artiste. Le renom et l'importance, cependant, nécessitent des écrits et du support institutionnel - par le biais d'archives d'expositions, de publications scientifiques, de bourses d'études, etc. De tels mécanismes ont été bien plus difficiles à obtenir pour des artistes en marge que pour ceux intégrés dans des métropoles géographiques et raciales. C'est ce qui fait des projets de recherche des artistes-chercheurs afro-américains Porter et Jones Pierre-Noël des études de cas importantes, et leurs archives des sites potentiellement fructueux pour de futurs contre-discours aux récits du milieu du xxe siècle sur l'art haïtien contemporain. 


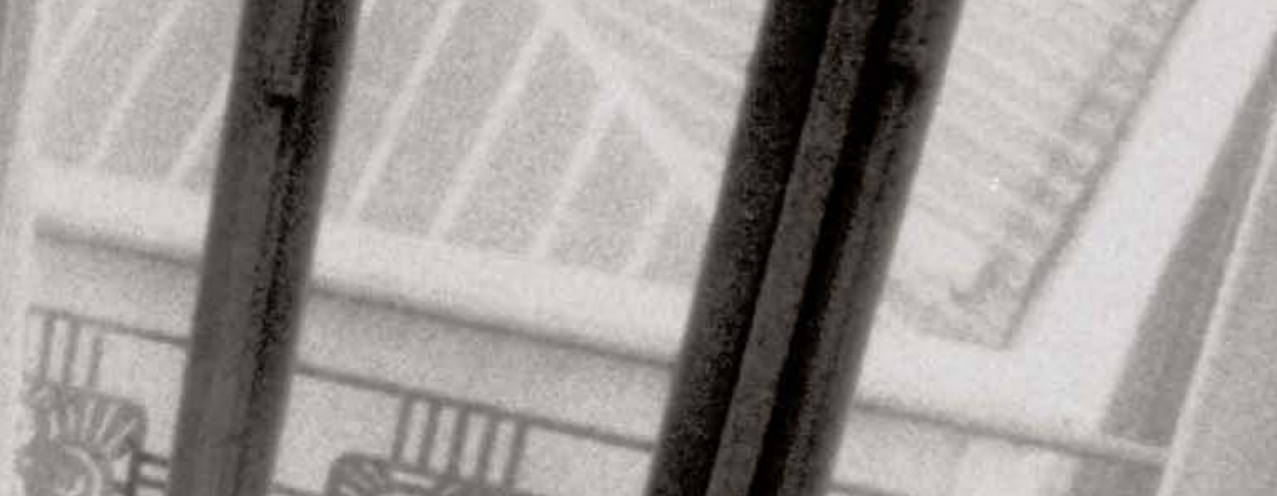
2) 1 stsin in $(939)$

151201 कर

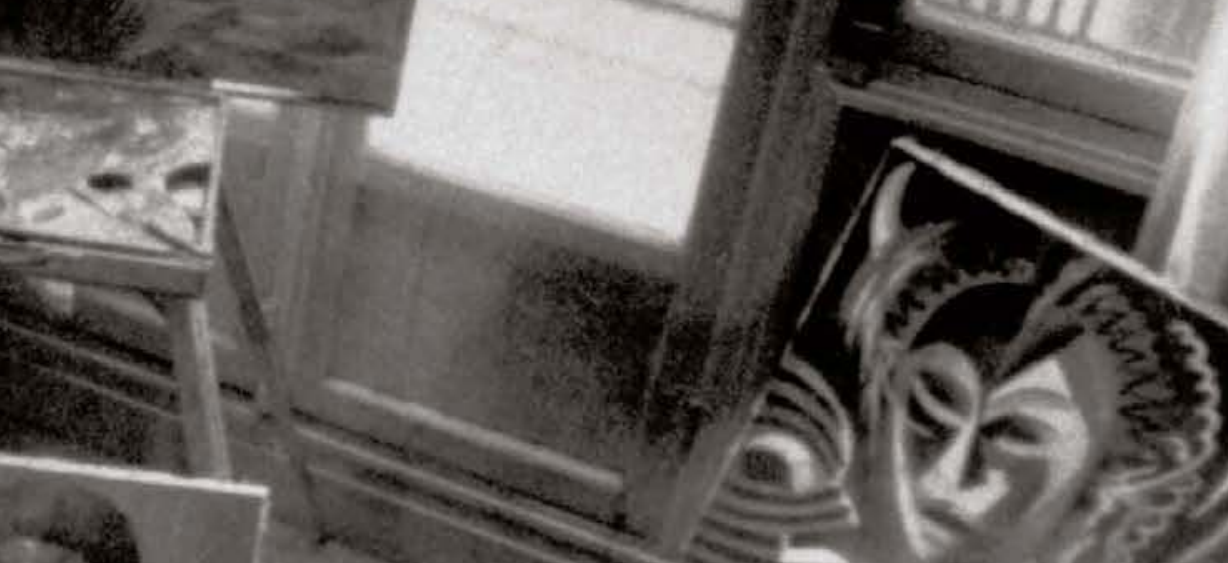
3is $1 / 250$

Wa is ifoth
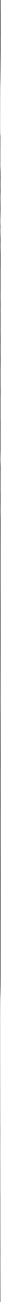